\title{
Detecção de cicatrizes de incêndios florestais utilizando a técnica de análise por vetor de mudança na terra indígena sete de setembro - Rondônia
}

\author{
Forest fire scars detection using change-vector analysis in the sete de \\ setembro indigenous land - Rondonia
}

Paulo Maurício Lima de Alencastro Graça' ${ }^{1 *}$

Claudia Suzanne Marie Nathalie Vitel'

Philip Martin Fearnside'

\section{Resumo}

A Terra Indígena Sete de Setembro (TISS) localiza-se no arco do desmatamento, caracterizada por ser uma região de grande pressão antrópica. Esta condição a torna susceptível às ameaças da degradação florestal por incêndios, exploração madeireira ilegal e corte raso para uso agropecuário. Assim, esse estudo teve por finalidade mapear áreas de floresta afetadas pela ação do fogo no período de 2010 a 2011, utilizando imagens TM/Landsat e a técnica de detecção por análise de vetor de mudança. Os resultados mostraram uma área total de 4.187 hectares de florestas afetadas pelo fogo no interior da TISS, correspondendo a cerca de 1,7 \% da área total da terra indígena. O monitoramento de terras indígenas por técnicas de sensoriamento remoto é fundamental para avaliar a vulnerabilidade dessas áreas às pressões humanas.

Palavras-chave: cicatriz de fogo; detecção de mudanças; terra indígena; sensoriamento remoto; Amazônia

\section{Abstract}

The Sete de Setembro Indigenous Land is situated in Brazil's "arc of deforestation," which is a region characterized by high anthropic pressure. This makes the reserve susceptible to forest degradation by fires, illegal logging and clearcutting for agriculture and ranching. The purpose of the present study was to map forest areas affected by fire from 2010 to 2011 using TM/Landsat images and the changevector analysis detection technique. The results showed a total area of 4187 hectares

I Instituto Nacional de Pesquisas da Amazônia - INPA; Endereço: Av. André Araújo, 2936, CEP: 69060-00I, Manaus, Amazonas, Brasil; E-mail: pmlag@inpa.gov.br (*) Autor para correspondência / claudia.vitel@gmail. com / pmfearn@inpa.gov.br 
of forest affected by fire in the indigenous reserve, or about $1.7 \%$ of the total reserve area. Monitoring of indigenous lands by remote sensing techniques is essential in order to evaluate the vulnerability of these areas to pressures from human activities.

Key words: Fire scar; change detection; indigenous land; remote sensing; Amazonia.

\section{Introdução}

As terras indígenas têm grande importância para a conservação da cobertura florestal na Amazônia. Pois, além de cobrirem extensas áreas florestais, as terras indígenas têm apresentado taxas de desmatamento baixas no interior dos seus limites em relação as suas áreas de amortecimento, assim como, em comparação ao resto da Amazônia brasileira. A conversão da cobertura florestal para atividades pecuárias e agrícolas na Amazônia tem reduzido os benefícios ambientais provenientes da floresta, tais como a proteção dos recursos hídricos, biodiversidade e a emissão evitada de gases de efeito estufa (GEEs), tanto localmente como em escala global (FEARNSIDE, 2006; MARENGO, 2007). Adicionalmente, nas últimas décadas, incêndios florestais originados de atividades humanas têm sido cada vez mais frequentes em florestas tropicais (ALENCAR et al., 2006; BROWN et al., 2006), contribuindo para o agravamento das emissões de GEEs e a perda de biodiversidade. Como alternativa a essas atividades convencionais estão sendo propostos para terras indígenas projetos de Redução de Emissões por Desmatamento e Degradação florestal (REDD), como é o caso do Projeto Carbono Suruí na Terra Indígena Sete de Setembro. No entanto, também os benefícios climáticos gerados por esse mecanismo podem ser ameaçados pela ocorrência de incêndios florestais. Desta maneira, a quantificação de perdas por incêndios florestais é importante para projetos de REDD, assim como é, também, para os inventários nacionais sob a Convenção Quadro das Nações Unidas sobre Mudança do Clima (UN-FCCC). Atualmente, não é obrigatório incluir as emissões dessa fonte, contabilizando-se apenas as emissões consideradas como "diretamente induzidas pelo homem"(IPCC, 2006). Para evitar que as concentrações alcancem um limite "perigoso", é necessário quantificar o total que está sendo emitido no mundo para que as cotas ("quantidades atribuídas") negociadas para os países sejam suficientes para limitar o aumento do total, não apenas o aumento da parte propositalmente emitida pela sociedade. Caso as emissões como as dos incêndios florestais não forem contabilizadas, então, as cotas negociadas serão insuficientes para conter o aquecimento global.

Neste contexto, o mapeamento da degradação florestal causada por incêndios florestais em áreas de proteção ambiental é de suma importância para se compreender vulnerabilidade dessas áreas às pressões humanas e para a quantificação das emissões de gases de efeito estufa. Este trabalho teve por objetivo mapear as áreas afetadas por incêndios florestais na Terra Indígena Sete de Setembro (TISS) utilizando a técnica de análise de vetor de mudança a partir de imagens Landsat-5 TM. 


\section{Material e Métodos}

\section{Área de Estudo}

A TISS possui $247.763^{2}$ hectares e se localiza na divisa entre os estados de Rondônia e Mato Grosso, abrangendo os municípios de Cacoal, Espigão D'Oeste e Rondolândia (Figura 1). A TISS foi identificada, registrada, homologada e oficialmente reconhecida pelo governo brasileiro através do decreto 88.867 de 18 de outubro de 1983, e é habitada por cerca de 1350 índios da etnia Paiter-Suruí, distribuídos em 21 aldeias. A cobertura vegetal dominante é de Floresta Ombrófila

2 Área estimada pelo Instituto Sócio-Ambiental a partir dos limites das terras indígenas publicados no Diário Oficial da União (http://ti.socioambiental.org/\#!/ terras-indigenas/3858)
Aberta com predominância de palmeiras e cipós, sob Argissolos Vermelho-Amarelos (RADAMBRASIL, 1978). A terra indígena está inserida na área de influência da rodovia BR-364, caracterizada por ser uma região de forte pressão de desmatamento conhecida como "Arco do desmatamento". As principais atividades econômicas são a pecuária bovina, a agricultura e a exploração madeireira.

\section{Descrição das Imagens}

Para a análise deste estudo foram utilizadas imagens ópticas do sensor TM Landsat-5, referentes às cenas 230/68 e 230/67 (Órbita/Ponto) de 20/07/ 2010 e 09/07/2011. As imagens utilizadas compreendem as coordenadas geográficas $61^{\circ} 32^{\prime} 11^{\prime \prime} \mathrm{W}$ e $60^{\circ} 42^{\prime}$ $27^{\prime \prime} \mathrm{W}$ de longitude e $10^{\circ} 42^{\prime} 22^{\prime \prime} \mathrm{S}$ e $11^{\circ} 24^{\prime} 11^{\prime \prime}$ $\mathrm{S}$ de latitude, e cobrindo uma área de 6.454,3 $\mathrm{km}^{2}$ (2872 x 2497 pixels).

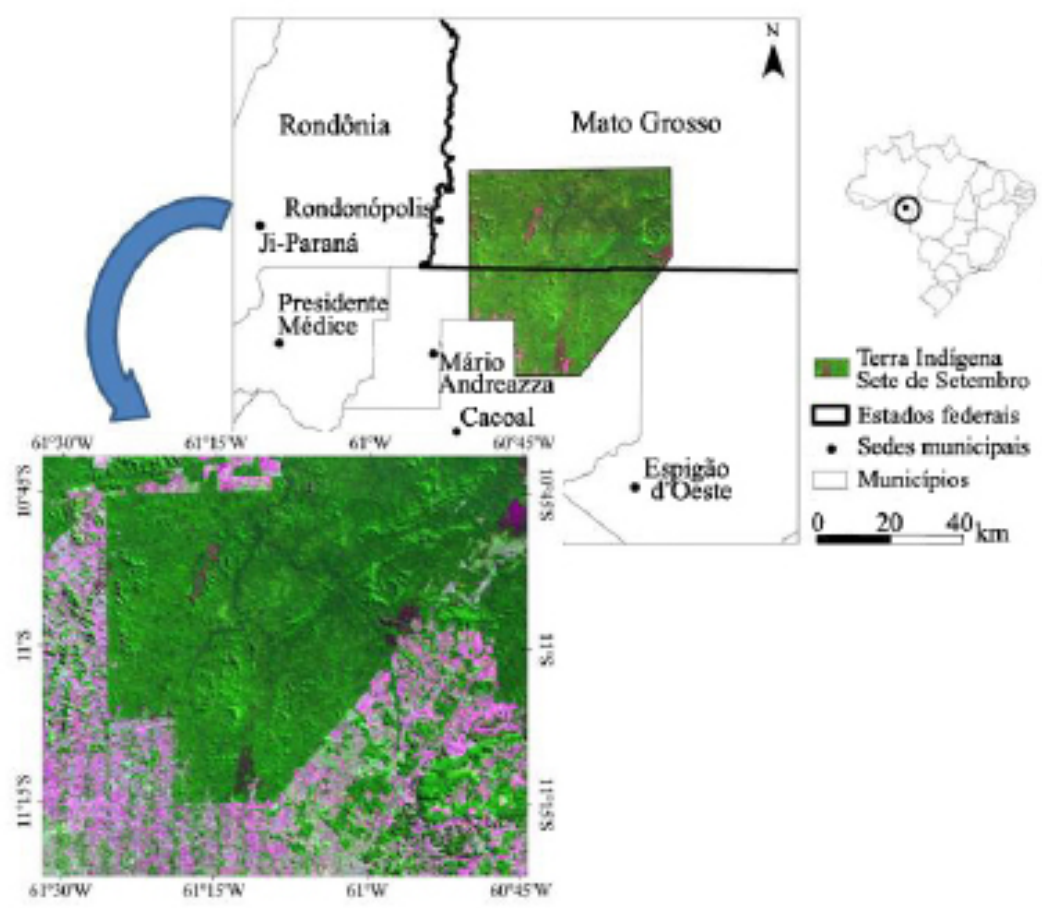

Figura I. Localização da área de estudo

Figure I.Study area location 


\section{Metodologia}

As etapas metodológicas utilizadas no mapeamento das cicatrizes de incêndios florestais neste estudo foram adaptadas de Graça et al. (2005) e se encontram esquematizadas na figura 2 .

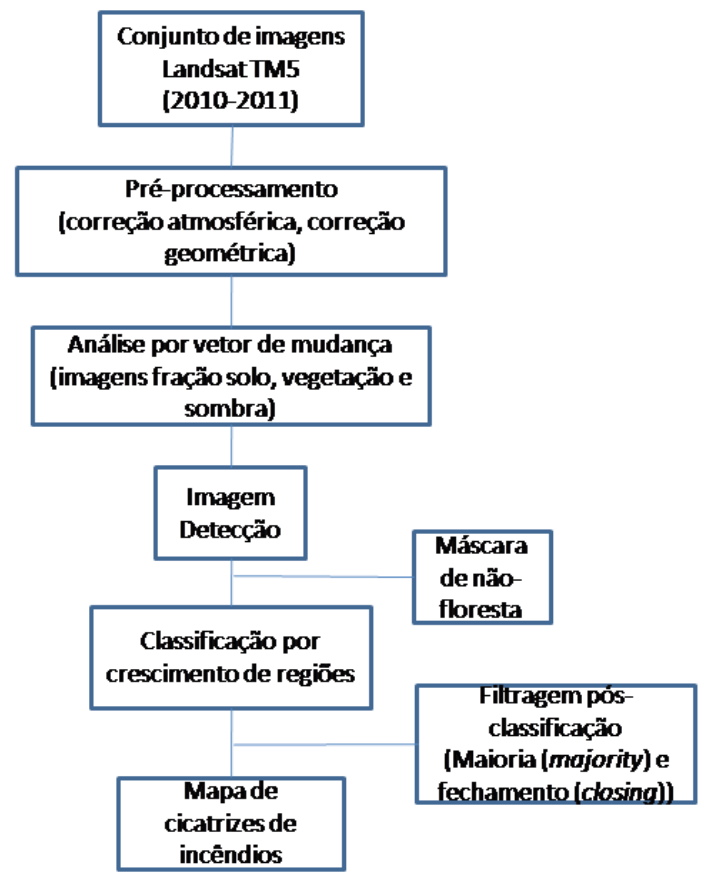

Figura 2. Fluxograma apresentado as etapas da metodologia utilizada

Figure 2. Flowchart showing the phases in the methodology.

\section{Pré-processamento}

As imagens utilizadas foram georreferenciadas a partir de imagens do banco de dados "geocover" da National Aeronautics and Space Administration (NASA), disponível na website $<$ https://zulu. ssc.nasa.gov/mrsid > para o par de anos 20102011, utilizando um modelo polinomial de primeiro grau e a reamostragem pelo método do vizinho mais próximo. $\mathrm{O}$ erro médio quadrático do registro das imagens foi menor do que 0,20 . O conjunto das imagens também foi corrigido para os efeitos da interferência atmosférica utilizando método automatizado do programa CLASlite (ASNER et al., 2009) que tem por base o modelo de transferência radiativa atmosférica 6S (VERMOTE et al.,1997) para derivar a refletância de superfície aparente para cada banda espectral. Por fim, as cenas 230/68 e 230/67 foram mosaicadas para as referidas datas.

\section{Modelo Linear de Mistura Espectral}

As imagens-fração solo, vegetação e sombra utilizadas na análise de vetor de mudança foram geradas a partir de um modelo linear de mistura espectral (BOARDMAN, 1989), conforme a equação 1 a seguir.

$r_{i}=\sum_{j=1}^{n} \rho_{i j} f_{j}+e_{i}$

onde,

$r_{i}$ reflectância de um dado pixel na banda espectral de $i$ n-bandas espectrais;

$n$ : número de componentes de mistura;

$\rho_{i j}$ reflectância do componente puro $j$ na banda espectral $i$;

$f_{j}$ : fração de cada componente puro $j$ dentro do pixel;

$e_{i}$ : expressa a diferença entre a reflectância do pixel observado $r_{i}$ e a reflectância do pixel computado a partir do modelo (termo residual).

Para a realização do modelo de mistura foram utilizados os componentes puros (endmembers) representativos das frações solo, vegetação e sombra. Os componentes puros foram obtidos diretamente das imagens com auxilio do algoritmo de índice de pureza de pixel (pixel purity índex - PPI) desenvolvido por Boardman et al. (1995). 


\section{Técnica de Detecção por Análise do Vetor de Mudança}

A análise de detecção de mudança por vetor de mudança utilizou a metodologia desenvolvida por Graça (2004). Esta técnica foi realizada a partir de um programa computacional desenvolvido na linguagem computacional Interactive Data Language (IDL), disponível no aplicativo ENVI. A análise foi procedida em 2 etapas: 1 ) Geração da imagem de magnitude do vetor de mudança (Equação 2) obtida a partir das diferenças resultantes da subtração entre as imagens frações solo, vegetação e sombra para o par de imagens (2010 vs. 2011);2) Geração de imagens Alfa e Beta obtidas a partir do ângulo formado pelo vetor de mudanças com os planos horizontal e vertical de um sistema de coordenadas esféricas, respectivamente (Figura 3). A magnitude indica a intensidade da mudança. Por sua vez, os ângulos alfa e beta determinam a direção do vetor de mudança dentro do limite da esfera. A equação 2 para calcular a magnitude do vetor de mudança é expressa por:

$$
\mu=\sqrt{(\Delta x)^{2}+(\Delta y)^{2}+(\Delta z)^{2}}
$$

onde,

$\mu$ é a magnitude do vetor de mudança; $\Delta x=x_{1}-x_{0} ; \Delta y=y_{1}-y_{0} ; \Delta z=z_{1}-z_{0} ;$

$\Delta \mathrm{x}$ é a diferença entre os valores das imagens fração solo da data inicial $\left(x_{0}\right)$ e final $\left(x_{1}\right)$; $\Delta y$ é a diferença entre os valores das imagens fração vegetação da data inicial $\left(y_{0}\right)$ e final $\left(y_{1}\right)$;

$\Delta z$ é a diferença entre os valores das imagens fração sombra da data inicial $\left(z_{0}\right)$ e final $\left(z_{1}\right)$;

A imagem Alfa $(\alpha)$ representada pelos valores dos ângulos horizontais formados pelo vetor de mudança e o plano originado

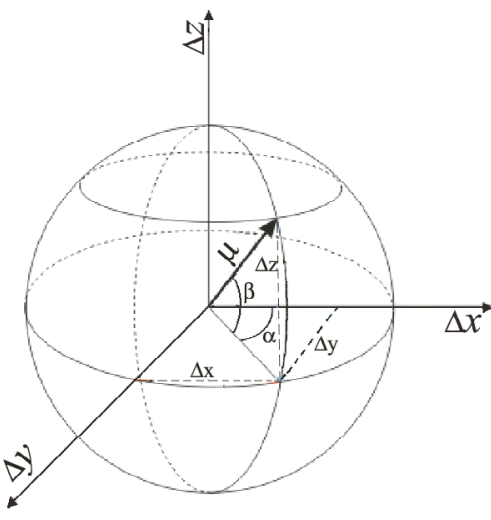

Figura 3. Representação do vetor de mudança $(\mu)$ em um sistema de coordenadas esféricas

Figure 3. Representation of the change vector $(\mu)$ in the spherical coordinate system

pelos eixos $\Delta \mathrm{x}$ (diferença entre os valores das frações solo) e $\Delta y$ (diferença entre os valores das frações vegetação) é obtida a partir da equação 3:

$\alpha=\operatorname{arctg}\left(\frac{\Delta y}{\Delta x}\right)$

Tal como em um sistema de coordenadas geográficas, o ângulo Alfa, representa a longitude da esfera e sua variação angular está compreendida entre $-180^{\circ}$ a $180^{\circ}$. No entanto, a equação (3) só permite gerar ângulos entre $-90^{\circ} \mathrm{e}$ $90^{\circ}$ correspondentes, respectivamente, ao primeiro e quarto quadrante, sob a suposição de que $\Delta x>0$. Desta maneira, para calcular o ângulo alfa para o segundo quadrante, quando $\Delta x<0$ e $\Delta y \geq 0$ utilizou-se a equação 4 . De forma análoga, para o terceiro quadrante foi utilizada a equação 5 para $\Delta x<0$ e $\Delta y<0$.

$\alpha=\operatorname{arctg}\left(\frac{\Delta y}{\Delta x}\right)+180^{\circ}$ 
$\alpha=\operatorname{arctg}\left(\frac{\Delta y}{\Delta x}\right)-180^{\circ}$

A imagem Beta $(\beta)$ é composta pelos valores dos ângulos verticais formados pelo vetor de mudança e o plano originado pelos eixos $\Delta \mathrm{x}$ (diferença entre os valores das frações solo) e $\Delta y$ (diferenças entre os valores das frações vegetação), conforme a figura 3. O ângulo Beta, que representa a latitude da esfera, foi calculado a partir da diferença entre os valores dos pixels da imagem fração de sombra $(\Delta z)$ em relação ao valor de magnitude, empregando a equação 6 . A sua variação angular está compreendida entre $-90^{\circ}$ a $90^{\circ}$.

$$
\beta=\operatorname{arcsen}\left(\frac{\Delta z}{\mu}\right)
$$

onde,

$\mu$ representa a magnitude do vetor de mudança.

A direção do vetor de mudança é caracterizada no sistema de coordenadas esféricas de acordo com as mudanças ocorridas entre a data inicial e final das imagens para as frações solo, vegetação e sombra, representando ganho ou perda das respectivas frações.

A partir das imagens magnitude, alfa e beta originadas da técnica Análise do Vetor de Mudança (AVM) foi gerada uma composição colorida $\mathrm{R}(\mu), \mathrm{G}(\alpha)$ e $\mathrm{B}(\beta)$, denominada "imagem de detecção de mudança".

\section{Máscara de Não Floresta}

Para melhorar o desempenho desta técnica, evitando interposição entre as respostas espectrais do solo em áreas desmatadas para atividade agropecuárias e aquelas originadas da exposição do solo em áreas afetadas por incêndios, foi criada uma máscara de não floresta. Esta foi originada a partir de uma classificação supervisionada de máxima verossimilhança utilizando-se todas as bandas ópticas da imagem adquirida em 2011.

\section{Classificação por Crescimento de Regiões}

A imagem detecção de mudanças resultante da AVM foi utilizada para o mapeamento de cicatrizes de incêndios florestais utilizando um classificador supervisionado de crescimento de regiões, desenvolvido em linguagem IDL. A classificação foi realizada em duas etapas. A primeira etapa teve por objetivo gerar as "sementes" para o crescimento de regiões, a partir pixels representativos de cicatrizes de incêndios na imagem detecção. A etapa seguinte teve por finalidade mapear as cicatrizes, a partir das sementes geradas na etapa anterior, utilizando um algoritmo de crescimento de regiões.

A primeira etapa utilizou um algoritmo de "semeadura" baseado em um filtro de janela móvel ( $4 \times 4$ pixels) para selecionar até quatro pixels (janela central de $2 \times 2$ pixels) considerados representativos de cicatrizes de incêndios na imagem deteç̧ão da AVM. Desta maneira, limiares mínimos para valores de magnitude $(0,35)$ e ângulos alfa $(-76,4)$ e beta $(-30,7)$ foram pré-estabelecidos para selecionar até quatro pixels centrais, a partir de amostras de pixels extraídos da própria imagem. Outros limiares de máximo para valores de magnitude $(0,53)$, alfa $(-30,2)$ e beta $(90,3)$, definidos a priori, foram aplicados aos doze pixels vizinhos da janela móvel, de modo a evitar a seleção de pixels centrais em áreas desmatadas tais como, estradas e solo exposto nas bordas da máscara de "não floresta", assim como em áreas de exploração 
florestal. Os valores dos limiares dos pixels centrais, valores mínimos e máximos, foram estabelecidos a partir de 150 amostras (pixels) selecionadas diretamente da imagem detecção, consideradas representativas de áreas de florestas afetadas pelo fogo, e os valores dos limiares dos pixels vizinhos a partir de 150 amostras de pixels vizinhos aos pixels considerados representativos de áreas de florestas queimadas. Um pixel foi selecionado para "semente" quando pelo menos um dos quatro pixels centrais apresentou valores de magnitude, alfa e beta maiores ou iguais aos limiares estabelecidos, $\mathrm{e}$ quando todos os pixels vizinhos apresentaram valores para magnitude, alfa e beta menores do que o limiar de vizinhança. Como resultado deste algoritmo foi gerada uma imagem binária (0-1), na qual os pixels identificados como "sementes" em potencial de áreas afetadas por fogo receberam o valor igual a um.

$\mathrm{Na}$ etapa final do procedimento de classificação, as áreas de floresta afetadas pelo fogo (cicatrizes) foram mapeadas utilizando um algoritmo de crescimento de região, implementado em IDL/ENVI. Este algoritmo é baseado em uma janela móvel (3x3 pixels), operando a partir de "sementes" identificadas na etapa anterior. $O$ processo de crescimento de região é inicializado quando o pixel central da janela encontra um pixel classificado com o valor igual a um (isto é, semente de cicatriz) que possua, em pelo menos um dos oito pixels vizinhos, o valor igual a zero (floresta). $\mathrm{O}$ algoritmo então analisa a vizinhança do pixel central a partir de um limiar máximo para alfa $(50,2)$ e beta $(40,1)$ e um limiar mínimo para a magnitude $(0,07)$. Valores baseados em 150 amostras extraídas da imagem detecção resultante. Apenas os pixels vizinhos com valores abaixo dos limiares máximos para alfa e beta, e com valor acima do mínimo para a magnitude, foram classificados como cicatrizes de incêndios na imagem binária. $\mathrm{O}$ algoritmo de crescimento também permite que um número de iterações seja estabelecido para repetir este processo até que todos os pixels sejam classificados satisfatoriamente, analisando-se visualmente a imagem resultante do processo.

$\mathrm{Na}$ etapa pós-classificação, a imagem classificada resultante foi submetida a um filtro de maioria $(3 \times 3)$ para a exclusão de pixels isolados e, posteriormente, aplicouse um filtro morfológico ( $3 \times 3$ pixels) para realizar uma operação de fechamento (closing), com a finalidade de preencher as lacunas originadas pelo algoritmo de crescimento e delimitar área de floresta afetada pelo fogo.

\section{Resultados e Discussão}

A técnica de detecção de análise por mudança de vetor utilizada neste estudo detectou uma área total de 9.815,94 hectares de cicatrizes de incêndios florestais. Deste total, $4.186,98$ ha $(42,7 \%)$ encontra-se no interior da terra indígena, correspondendo a $1,7 \%$ da área total da Terra Indígena Sete de Setembro. Observou-se neste estudo que em algumas áreas de incêndio florestal de baixa intensidade, o algoritmo de "semeadura" não conseguiu selecionar pixels potenciais para o uso na etapa de classificação por crescimento de regiões, impossibilitando o mapeamento destas áreas. Para contornar esse problema foi realizada a edição manual de sementes na imagem para que o classificador de crescimento de regiões pudesse mapear estas áreas. Incêndios florestais nem sempre atingem a copa das árvores, em geral, quando a floresta possui o 
dossel fechado, ele ocorre de forma branda na superfície do piso florestal (COCHRANE; SCHULZE, 1999). Assim, áreas de floresta primária e florestas exploradas muito antigas (com o dossel regenerado por espécies do processo secundário) podem apresentar uma degradação branda pela ação do fogo, difícil de ser percebida pela técnica de detecção de mudanças em imagens de satélite. Em florestas exploradas com dossel desestruturado, o fogo age da mesma maneira do que nas florestas intactas, porém a ação do fogo pode ser muito mais intensa devido a grande quantidade de resíduos vegetais (material combustível) deixados pelo processo de extração madeireira (UHL; KAUFFMAN, 1990; GERWING, 2002). Uma avaliação estatística do desempenho desta técnica no mapeamento de cicatrizes de incêndio gerou um Kappa condicional igual a $\hat{k i+}=0,97$ (exatidão do usuário) e $\hat{k}$ $\mathrm{j}+=0,85$ (exatidão do produtor). De acordo com o relato de índios que habitam o lugar, a origem provável do incêndio, observado na figura 4, foi causada pela propagação acidental do fogo de uma área de pastagem vizinha à floresta.
A grande ocorrência de incêndios dentro da terra indígena verificada nesse estudo pode estar relacionada ao evento extremo de seca ocorrido em 2010. Neste ano a precipitação pluviométrica na estação seca foi baixa na Amazônia (LEWIS et al., 2011), aumentando o risco de ocorrência de incêndios florestais.

A terra indígena Sete de Setembro tem sido alvo de atividade madeireira ilegal desde meados da década de 80 (FERRONATO; NUNES, 2011). Recentemente, com o empobrecimento dos recursos madeireiros, a população dos Paiter-Surui tem convertido as áreas de floresta para a introdução das atividades de pecuária e cafeicultura, como alternativa econômica. A conversão de floresta para atividades agrícolas e pecuária é realizada com a prática agrícola de corte e queima, colocando em risco a floresta remanescente aos incêndios. Esses motivos explicam, também, o aumento do desmatamento na TISS na última década.

Para evitar o desmatamento na TISS foi proposto aos índios Paiter-Suruí um projeto de conservação, denominado Projeto de Carbono Florestal Suruí (IDESAM, 2011).
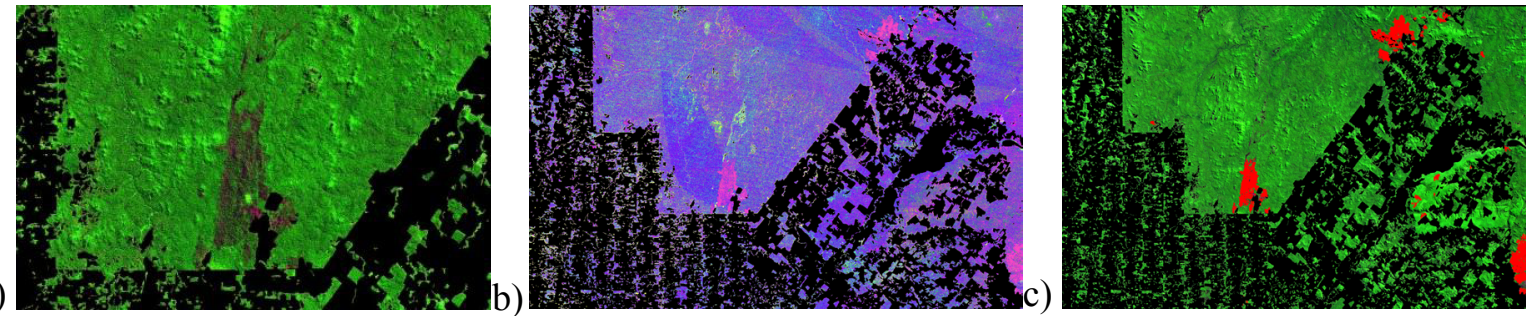

Figura 4. Recorte da imagem da área de estudo evidenciando cicatrizes de incêndio florestal na parte sul daTISS. a) imagem Landsat $R(5), G(4), B(3)$ do ano de 201 I; b) imagem detecção $R$ (Magnitude),G(Alfa) e B(Beta); e c) cicatriz de fogo mapeada em vermelho sob a imagem (a). As áreas em preto correspondem à máscara de não floresta.

Figure 4. Subsample of the study area image depicting the indigenous land area with forest fire scars: a) Landsat 201 I image $R(5), G(4), B(3)$; b) detection image R(Magnitude),G(Alfa) e $\mathrm{B}($ Beta); and c) mapped fire scars indicated in red under the (a) image. Black areas corresponds to the non-forest mask. 
Esse projeto utiliza o mecanismo de Redução de Emissões por Desmatamento e Degradação florestal (REDD), que é conceitualmente baseado no pagamento de serviços ambientais para manter as florestas em pé. No entanto, a compensação do serviço ambiental da floresta em evitar a emissão de gases de efeito estufa por meio deste mecanismo tem sido criticada por não serem permanentes, ou seja, podem ocorrer eventos não controlados, tais como incêndios florestais, que causam perda de parte do estoque de carbono e, portanto, reduzem os benefícios climáticos da floresta. Este fato pode levar a se premiar as emissões evitadas que não são permanentes. $\mathrm{O}$ risco de incêndios em áreas de floresta mantidas em projetos de REDD foi previsto por Aragão e Shimabukuro (2010). O presente trabalho demonstra que incêndios de fato ocorrem nessas áreas, e que é possível mensurar as suas dimensões por meio de sensoriamento remoto.

A falta de "permanência eterna" do carbono na floresta não significa, no entanto, que projetos que mantem carbono fora da atmosfera de forma temporária não tenham valor climático, embora esse valor, evidentemente, é menor que uma emissão evitada que fosse permanente (FEARNSIDE, 2002). A questão do tratamento do valor do tempo na contabilidade do carbono é um dos principais itens na "batalha teórica" que cerca o assunto dos serviços ambientais da floresta amazônica (FEARNSIDE, 2012). Monitoramento e quantificação de perdas por incêndios florestais são essenciais para ter contabilidade e premiação justas dos serviços ambientais de áreas como estas do Projeto Carbono Suruí na Terra Indígena Sete de Setembro.

\section{Conclusões}

A metodologia aplicada neste estudo demonstrou que ocorrência de incêndios florestais na TISS teve grande extensão (4,2 mil ha). Incêndios florestais causam impactos ambientais graves e podem reduzir os benefícios climáticos gerados pela manutenção da floresta em pé. $\mathrm{O}$ monitoramento de terras indígenas por técnicas de sensoriamento remoto é fundamental para avaliar a vulnerabilidade dessas áreas às pressões humanas.

\section{Agradecimentos}

Agradecemos ao Instituto Nacional de Ciência e Tecnologia dos Serviços Ambientais da Amazônia (Inct-Servamb), CNPq (processo no: $573810 / 2008-7$ ), pelo suporte financeiro

\section{Referências}

ARAGÃO, L. E. O. C.; SHIMABUKURO, Y. E. The Incidence of Fire in Amazonian Forests with Implications for REDD. Science, v.328, p.1275-1278, 2010.

ASNER, G. P.; KNAPP, D. E.; BALAJI, A.; PÁEZ-ACOSTA, G. Automated mapping of tropical deforestation and forest degradation: CLASlite. Journal of Applied Remote Sensing, v.3, n.3, p.35-43, 2009. 
ALENCAR, A.;NEPSTAD, D.; VERA DIAZ, M.C. Forest understory fire in the Brazilian Amazon in ENSO and non-ENSO years: Area burned and committed carbon emissions. Earth Interactions. v.10, n.6, p.1-17, 2006.

BARLOW,J.; PERES, C. A.; LAGAN, B.; HAUGAASEN, T. Large tree mortality and the decline of forest biomass following Amazonian wildfires. Ecology Letters, v.6, p.6-8, 2003.

BOARDMAN, J. W. Inversion of imaging spectrometry data using singular value decomposition. In: INTERNATIONAL GEOSCIENCE AND REMOTE SENSING SYMPOSIUM (IGARSS'89). 12., 1989. Vancouver, Proceedings of Canadian Symposium on Remote Sensing, 1989. Editado por IEEE, 1989. v.4, p.2069-2072.

BROWN,F.; SCHROEDER,W.; SETZER,A.W.; MALDONADO,M.L.R.;PANDOJA, N.; DUARTE, A.; MARENGO, J. A. Monitoring fires in Southwestern Amazonia rain forest.EOS, Transactions of the American Geophysical Union, v.87, n.26, p.253-264, 2006.

COCHRANE, M.A.; SCHULZE. M.D. Fire as a recurrent event in tropical forests of the eastern Amazon: effects on forest structure, biomass, and species composition. Biotropica. v.31, p. 2-16, 1999.

FEARNSIDE, P.M.Time preference in global warming calculations: A proposal for a unified index. Ecological Economics, v. 41, n.1, p. 21-31, 2002

FEARNSIDE, P. M. Desmatamento na Amazônia: dinâmica, impactos e controle. Acta Amazonica, v.36, n.3, p. 395-400, 2006.

FEARNSIDE, P.M. The theoretical battlefield: Accounting for the climate benefits of maintaining Brazil's Amazon forest. Carbon Management, v.3, n.2, p. 145-148. 2012.

FERRONATO, M.L.; NUNES, R.O. A exploração ilegal de madeiras na terra indígena Sete de Setembro, Cacoal - RO. Revista Científica FACIMED, v.3, n.3, p.1-11, 2011.

GERWING, J. J. Degradation of forests through logging and fire in the eastern Brazilian Amazon. Forest Ecology and Management, v.157, p.131-141, 2002.

GRAÇA, P. M. L. A.; SANTOS, J. R.; SOARES, J. V.; SOUZA, P. E. U. Desenvolvimento metodológico para detecção e mapeamento de áreas florestais sob exploração madeireira: estudo de caso, região norte do Mato Grosso.In: SIMPÓSIO BRASILEIRO DE SENSORIAMENTO REMOTO, 12., 2005.Goiânia. Anais... Goiânia, INPE, 2005, p.1555-1562.

IDESAM. Projeto de Carbono Florestal Suruí. Instituto de Conservação e Desenvolvimento Sustentável do Amazonas - IDESAM, Manaus, 2011.129p.

INSTITUTO SOCIOAMBIENTAL (ISA), De Olho nas Terras Indígenas no Brasil. Disponível em: <http://ti.socioambiental.org/\#!/destaque/desmatamento >. Acesso em: 10 jun. 2012. 
IPCC. 2006 IPCC Guidelines for National Greenhouse Gas Inventories. Prepared by the National Greenhouse Gas Inventories Programme, EGGLESTON, H.S.; BUENDIA, L.; MIWA, K.; NGARA, T.; TANABE, K. (Eds.). Intergovernmental Panel on Climate Change (IPCC) \& Institute for Global Environmental Strategies (IGES), Kanagawa, Japão, 2006.

LEWIS, S. N. L.; BRANDO, P. M.; PHILLIPS, O. L.; VAN DER HEIJDEN, G. M. F.; NEPSTAD, D. The 2010 Amazon Drought. Science, v. 331, n. 6017, p. 554, 2011.

MARENGO, J. A. Mudanças climáticas globais e seus efeitos sobre a biodiversidade: caracterização do clima atual e definição das alterações climáticas para o território brasileiro ao longo do século XXI. 2. ed. Brasília: MMA, 2007.212p.

UHL, C.; KAUFFMAN, J. B. Deforestation, fire susceptibility, and potential tree responses to fire in the eastern Amazon. Ecology, v71, p.437-449, 1990.

VERÍSSIMO, A.; ROLLA, A.; VEDOVETO, M.; FUTADA, S. M. Áreas Protegidas na Amazônia Brasileira: avanços e desafios. Belém/São Paulo: Imazon e ISA. 2011. p.87.

VERMOTE, E. F.; TANRE, D.; DEUZE, J. L.; HERMAN, M.; MORCRETTE, J.J. S. Second Simulation of the Satellite Signal in the Solar Spectrum, 6S: An Overview. IEEE Transactions On Geoscience And Remote Sensing, v.12, n. 3, p. 675-686, 1997. 